\title{
Numerical and experimental approach for identifying elastic parameters in sandwich plates
}

\author{
Sergio Ferreira Bastos ${ }^{\mathrm{a}}$, Lavinia Borges ${ }^{\mathrm{b}}$ and Fernando A. Rochinha ${ }^{\mathrm{b}, *}$ \\ a SENAI - CETIQT, Rua Dr. Manoel Cotrim 195, Riachuelo, Rio de Janeiro, RJ, Brazil \\ E-mail: sbastos@cetiqt.senai.br \\ ${ }^{\mathrm{b}}$ LMS - Solid Mechanics Laboratory, Mechanical Engineering Department, Federal University of Rio de Janeiro, \\ C.P. 68503, Rio de Janeiro, RJ, Brazil \\ E-mail: lavinia@ufrj.br;faro@serv.com.ufrj.br
}

\begin{abstract}
This article deals with the identification of elastic parameters (engineering constants) in sandwich honeycomb orthotropic rectangular plates. A non-destructive method is introduced to identify the elastic parameters through the experimental measurements of natural frequencies of a plate undergoing free vibrations. Four elastic constant are identified. The estimation of the elastic parameter problem is solved by minimizing the differences between the measured and the calculated natural frequencies. The numerical method to calculate the natural frequencies involves the formulation of Rayleigh-Ritz using a series of characteristic orthogonal polynomials to properly model the free edge boundary conditions. The analysis of the results indicates the efficiency of the method.
\end{abstract}

Keywords: Identification of parameters, sandwich plates, optimization

\section{Introduction}

Nowadays, composite structural systems are extensively adopted as solutions to many engineering applications such as automobiles, railways or aerospace vehicles. This situation is a direct consequence of their superior characteristics in the structural response.

Elastic parameters, like stiffness or the Poisson's ratio, play a crucial role in analyzing, designing, manufacturing and controlling structural systems. The conventional techniques of identification of those parameters relies on static tests which do not seem to be adequate for non conventional materials or structures as, for instance, laminates or any general sort of composites. They often present an orthotropic or transversal isotropic behavior and thus require more reliable and

\footnotetext{
${ }^{*}$ Corresponding author.
}

robust identification approaches that lead to the estimation of all involved constants. Besides, those materials present a great dispersion on their behavior when different specimens are observed, at least when they are compared to standard materials. This demands the execution of several experiments in order to obtain more reliable results. Therefore, cheap, simple and non destructive experiments are mandatory.

Motivated by this, a methodology for the estimation of elastic constants involving modal analysis combined with optimization processes is presented here. This approach fits in the so called model up-date which seeks matching experimental results to analytical modeling. The proposed technique has turned out to be appropriated for composites and was applied here to a honeycomb sandwich plate with aluminum core and face sheets.

In the present research, laminated plates have been chosen as basic structure for identification [1]. Those 
structures are submitted to standard modal tests, from which natural frequencies are obtained. In the second step of the estimation, those experimental results are compared to their counterparts obtained from analytical models. Hence an optimization algorithm is used to fit those analytical models in order to yield the sought elastic constants. This approach was assessed by means of a number of different situations and has come to very satisfactory results.

Indeed, similar approaches have been used recently and they are extensively presented in literature [3,5-8, $10,11]$. In most of the cases the classical plate theory is employed and natural frequencies are used as basic information to the identification process. Although, there are several and crucial different aspects among them, like, for instance, the numerical algorithms, the type of experiments, the adopted boundary conditions (supports of the plates) and the way used to generate the discrete model. Anyway, the good results obtained by all those approaches represent an additional motivation to explore more and more those techniques in order to complement or substitute conventional static tests in the determination of material constants. Nevertheless, no one of the above mentioned authors deal with the identification of elastic constants for sandwich panels using, simultaneously, optimization approach, modal analysis and plate theory. Saito et al. [11] adopted the optimization approach but the theoretical model is based on orthotropic Timoshenko beam theory, so they could only identify two elastic parameters.

\section{Experimental analysis [1]}

A standard modal test has several different ways to be performed. They differ in how the structure is supported, what the excitation sources are and how the response is measured. Here, a plate was chosen as the basic structure, as previously mentioned. For simulating a free structure, the plate was hanged by two nylon wires and impact tests were carried out. Due to the experimental simplifications, accelerations of some points were used as the structural response. A more detailed description of the experimental set-up comes bellow.

The tested sandwich plate was formed by two thin sheets of aluminum with $0.7 \mathrm{~mm}$ of thickness and a honeycomb core whose dimensions were: $0.60 \mathrm{~m} \times$ $0.40 \mathrm{~m} \times 0.0105 \mathrm{~m}$ and the mass was $0.725 \mathrm{~kg}$. As mentioned before the plate was suspended by two nylon wires attached to it by means of very small sup- ports. Screws in the wall connected those wires and were used to maintain the correct position of the plate. A small impact hammer was used to excite the structure. Accelerometers attached to the plate were linked by signal conditioners to an HP 3566a data signal analyzer which, in turn, was controlled by a personal computer. The situation is presented, in a schematic way, in Fig. 1 and a basic experiment configuration is depicted in Fig. 2.

In order to obtain sufficient data for the identification process, three sets of tests with different positions of one accelerometer were performed $((110,120)$; (250,140); $(330,260) \mathrm{mm}$ coordinates were established from the left upper corner). Each set corresponds to the average of twenty tests with the aim of minimizing the presence of noise in the data.

A modal analysis code, developed by the Vibration Laboratory of PUC-Rio, was used to post-process data. This consists on curve fitting the measurements with the Frequency Response Function (FRF). Only the natural frequencies were extracted from the FRF because a full modal analysis leading to the mode shapes would have required much more time and computational effort. Therefore, a careful ad-hoc analysis necessary to assure the correct correlation among experimental and numerical frequencies was needed.

\section{Mathematical modeling}

The analytical discrete model used in the identification process is based on a variational framework namely the Rayleigh-Ritz method, leading to approximate solutions for the mode shapes and natural frequencies. The modeling considers the composite structure formed by a central core and the two face sheets as an homogenous plate with global equivalent constitutive constants, which have to be obtained by means of analytical or experimental techniques.

The adopted kinematics comes from the Mindlin plate theory and it is described by the three fields $w_{0}, \psi_{x}$ and $\psi_{y}$. The first one is the vertical displacement of mid-surface points and the last two corresponds to the rotation of the cross sections in $x$ and $y$ directions. Here $x$ and $y$ stand for orthogonal directions in the midsurface of the plate. In this theory, plane cross-sections remain plane during the motion and shear deformation is accomplished by considering the rigid rotation of the cross-sections, given by $\psi_{x}$ and $\psi_{y}$.

Therefore, assuming the plate to be a conservative system experimenting a free harmonical motion corre- 


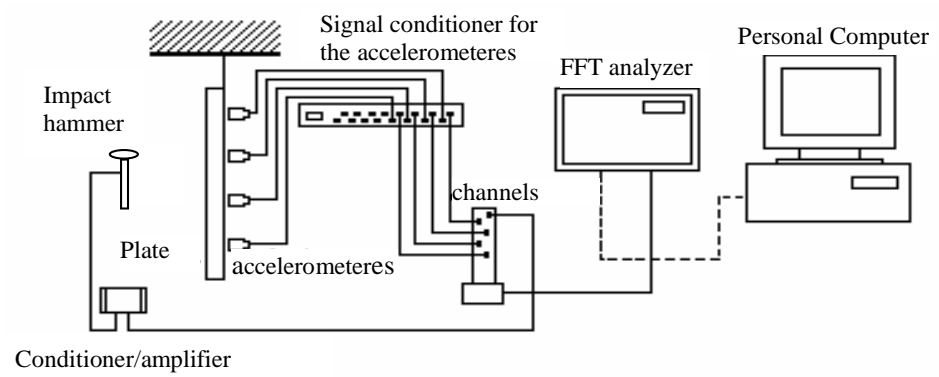

Fig. 1. The scheme of the experimental set-up.

sponding to a specific vibration mode, the accumulated energy in a specific instant is given by $[9,11]$

$$
\begin{aligned}
& T_{\max }= \\
& \omega^{2}\left(\begin{array}{l}
\frac{1}{24}\left(6 \rho_{f} c^{2} t+\rho_{c} c^{3}\right) \int_{A}\left(\psi_{x}^{2}+\psi_{y}^{2}\right) d A \\
+\frac{1}{2}\left(2 \rho_{f} t+\rho_{c} c\right) \int_{A} w_{0}^{2} d A
\end{array}\right) \\
& U_{\max }=\frac{E c^{2} t}{4\left(1-\nu^{2}\right)} \int_{A}\left(\begin{array}{l}
\psi_{x, x}^{2}+\psi_{y, y}^{2} \\
+2 \nu \psi_{x, x} \psi_{y, y}
\end{array}\right) d A \\
& \quad+\frac{E c^{2} t}{8(1+\nu)} \int_{A}\left(\psi_{x, y}+\psi_{y, x}\right)^{2} d A \\
& \quad+\frac{c}{2} k G_{y z} \int_{A}\left(w_{0, y}-\psi_{y}\right)^{2} d A \\
& \quad+\frac{c}{2} k G_{z x} \int_{A}\left(w_{0, x}-\psi_{x}\right)^{2} d A .
\end{aligned}
$$

where $T$ and $U$ stand for kinetic and elastic energy terms. Besides, $\omega$ is the natural frequency, $\rho_{f}$ and $\rho_{c}$ are, respectively, the mass density of the faces and of the core, $\nu$ stands for the Poisson's ratio, $E$ and $G_{z x}, G_{y z}$ are the moduli of elasticity and shear. Finally, $k$ is the shear correction factor and a typical value for it is 0.8 [9]. The constitutive parameters $E, G_{z x}, G_{y z}$ and $\nu$ are the target of the identification process. The notation $f,{ }_{x}$ denotes spatial derivatives in $x$ or $y$ directions.

The dimensions of the plate's cross section with area A are introduced in Fig. 3.

The three kinematics fields were discretized by using orthogonal polynomials which naturally enforce the free boundary conditions. In order to come up with simpler expressions the following non-dimensional coordinates are introduced

$$
\xi=\frac{2 x}{a} \quad \eta=\frac{2 y}{b}
$$

where $\xi$ e $\eta$ in $[-1,1]$ and $a$ and $b$ are the plate dimensions in directions $x$ and $y$.

Therefore, the displacement fields are now represented by

$$
\begin{aligned}
& w_{0}(\xi, \eta)=\sum_{m=0}^{p-1} \sum_{n=0}^{p-1} N_{m n} \phi_{m}(\xi) \phi_{n}(\eta) \\
& \psi_{x}(\xi, \eta)=\sum_{m=0}^{p-1} \sum_{n=0}^{p-1} O_{m n} \phi_{m}(\xi) \phi_{n}(\eta) \\
& \psi_{y}(\xi, \eta)=\sum_{m=0}^{p-1} \sum_{n=0}^{p-1} P_{m n} \phi_{m}(\xi) \phi_{n}(\eta)
\end{aligned}
$$

where $N, O$ and $P$ are the coefficients of the discretization, the integer $p$ corresponds to the number of adopted polynomials. Besides, $\phi_{m}$ and $\phi_{n}$ are the orthogonal polynomials referred above and defined by [4]:

$$
\begin{aligned}
& \phi_{0}(\xi)=\frac{1}{\sqrt{2}} \quad \phi_{i}=A_{i}^{-1 / 2} \Phi_{i}(\xi) \\
& \Phi_{1}=\xi \phi_{0} \quad \Phi_{i}=\xi \phi_{i-1}(\xi)-A_{i-1}^{1 / 2} \phi_{i-2}(\xi) \\
& (i=2,3,4, \ldots) \quad A_{i}=\int_{-1}^{1} \Phi_{i}^{2}(\xi) d \xi
\end{aligned}
$$

Substituting Eq. (4) in Eq. (2) leads to

$$
\begin{aligned}
& T_{\max }= \\
& \omega^{2} \frac{a b}{4}\left(\begin{array}{c}
\frac{1}{24}\left(\begin{array}{c}
6 \rho_{f} c^{2} t \\
+\rho_{c} c^{3}
\end{array}\right) \int_{-1}^{1} \int_{-1}^{1}\left(\begin{array}{c}
\psi_{x}^{2} \\
+\psi_{y}^{2}
\end{array}\right) d \xi d \eta \\
+\frac{1}{2}\left(2 \rho_{f} t+\rho_{c} c\right) \int_{-1}^{1} \int_{-1}^{1} w_{0}^{2} d \xi d \eta
\end{array}\right) \\
& U_{\max }=U_{\max }^{(1)}+U_{\max }^{(2)}+U_{\max }^{(3)}+U_{\max }^{(4)}
\end{aligned}
$$

where

$$
\begin{aligned}
U_{\max }^{(1)}= & \frac{E c^{2} t}{4\left(1-\nu^{2}\right)}\left[\frac{b}{a} \int_{-1}^{1} \int_{-1}^{1} \psi_{x, \xi}^{2} d \xi d \eta\right. \\
& +\frac{a}{b} \int_{-1}^{1} \int_{-1}^{1} \psi_{y, \eta}^{2} d \xi d \eta \\
& \left.+2 \nu \int_{-1}^{1} \int_{-1}^{1} \psi_{x, \xi} \psi_{y, \eta} d \xi d \eta\right]
\end{aligned}
$$




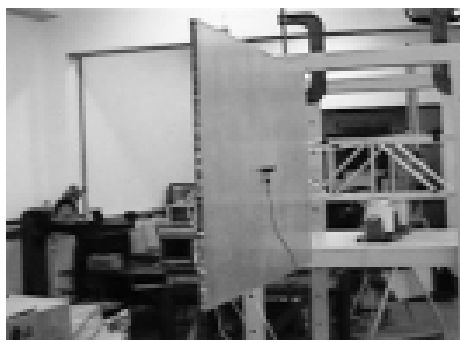

Suspended Plate

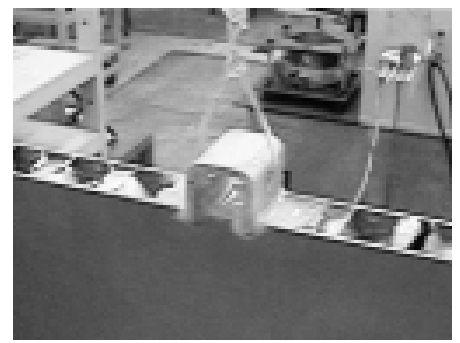

Support details

Fig. 2. Experimental configuration.

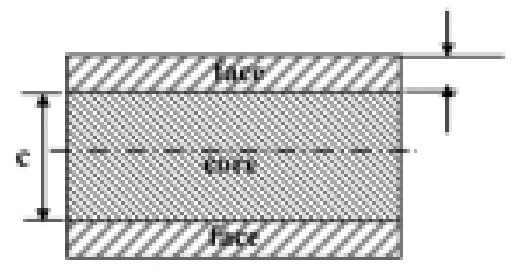

Fig. 3. Cross section dimensions.

$$
\begin{aligned}
U_{\max }^{(2)}= & \frac{E c^{2} t}{8(1+\nu)}\left[\frac{a}{b} \int_{-1}^{1} \int_{-1}^{1} \psi_{x, \eta}^{2} d \xi d \eta\right. \\
& +2 \int_{-1}^{1} \int_{-1}^{1} \psi_{x, \eta} \psi_{y, \xi} d \xi d \eta \\
& \left.+\frac{b}{a} \int_{-1}^{1} \int_{-1}^{1} \psi_{y, \xi}^{2} d \xi d \eta\right] \\
U_{\max }^{(3)}= & \frac{c}{2} G_{y z}\left[\frac{a}{b} \int_{-1}^{1} \int_{-1}^{1} w_{0, \eta}^{2} d \xi d \eta\right. \\
& -a \int_{-1}^{1} \int_{-1}^{1} w_{0, \eta} \psi_{y} d \xi d \eta \\
& \left.+\frac{a b}{4} \int_{-1}^{1} \int_{-1}^{1} \psi_{y}^{2} d \xi d \eta\right] \\
& \frac{c}{2} G_{z x}\left[\frac{b}{a} \int_{-1}^{1} \int_{-1}^{1} w_{0, \xi}^{2} d \xi d \eta\right. \\
& -b \int_{-1}^{1} \int_{-1}^{1} w_{0, \xi} \psi_{x} d \xi d \eta \\
& \left.+\frac{a b}{4} \int_{-1}^{1} \int_{-1}^{1} \psi_{x}^{2} d \xi d \eta\right] .
\end{aligned}
$$

The dynamical equilibrium state of the system is given by a minimum of the Lagrangian $L=T-V[4$, 9], therefore it satisfies

$$
\frac{\partial\left(T_{\max }-U_{\max }\right)}{\partial\left(N_{i j}, O_{i j}, P_{i j}\right)}=0 .
$$

Relation Eq. (9) leads to the following system of equations, which involves the coefficients $N, O$ and $P$

$$
\left\{\begin{array}{l}
\tilde{\tilde{\rho}} \tilde{f} \tilde{f}^{2} \sum_{m=0}^{p-1} \sum_{n=0}^{p-1} H_{m i n j}^{0000} N_{m n}=\sum_{m=0}^{p-1} \sum_{n=0}^{p-1} C_{m i n j}^{N N} N_{m n} \\
+\sum_{m=0}^{p-1} \sum_{n=0}^{p-1} C_{m i n j}^{N O} O_{m n}+\sum_{m=0}^{p-1} \sum_{n=0}^{p-1} C_{m i n j}^{N P} P_{m n} \\
\tilde{f}^{2} \sum_{m=0}^{p-1} \sum_{n=0}^{p-1} H_{m i n j}^{0000} O_{m n}=\sum_{m=0}^{p-1} \sum_{n=0}^{p-1} C_{m i n j}^{O N} N_{m n} \\
+\sum_{m=0}^{p-1} \sum_{n=0}^{p-1} C_{m i n j}^{O O} O_{m n}+\sum_{m=0}^{p-1} \sum_{n=0}^{p-1} C_{m i n j}^{O P} P_{m n} \\
\tilde{f}^{2} \sum_{m=0}^{p-1} \sum_{n=0}^{p-1} H_{m i n j}^{0000} P_{m n}=\sum_{m=0}^{p-1} \sum_{n=0}^{p-1} C_{m i n j}^{P N} N_{m n} \\
+\sum_{m=0}^{p-1} \sum_{n=0}^{p-1} C_{m i n j}^{P O} O_{m n}+\sum_{m=0}^{p-1} \sum_{n=0}^{p-1} C_{m i n j}^{P P} P_{m n}
\end{array}\right.
$$

where

$$
\begin{aligned}
H_{m i n j}^{\alpha \beta \delta \varepsilon}= & \int_{-1}^{1} \int_{-1}^{1}\left[\frac{d^{\alpha} \phi_{m}(\xi)}{d^{\alpha} \xi} \frac{d^{\beta} \phi_{i}(\xi)}{d^{\beta} \xi}\right. \\
& \frac{d^{\delta} \phi_{j}(\eta)}{d^{\delta} \eta} \frac{d^{\varepsilon} \phi_{n}(\eta)}{d^{\varepsilon} \eta} d \xi d \eta \\
C_{\text {minj }}^{N N}= & \frac{\tilde{\rho}}{\tilde{I}} \\
& \left(\frac{a^{2}}{b^{2} \pi^{2}} \frac{\bar{G}_{y z}}{E} H_{\text {minj }}^{0011}+\frac{1}{\pi^{2}} \frac{\bar{G}_{z x}}{E} H_{\text {minj }}^{0001}\right) \\
C_{\text {minj }}^{N O}= & -\frac{\tilde{\rho}}{\tilde{I}} \frac{a}{2 \pi^{2}} \frac{\bar{G}_{z x}}{E} H_{m i n j}^{0100} \\
C_{m i n j}^{N P}= & -\frac{\tilde{\rho}}{\tilde{I}} \frac{a^{2}}{2 b \pi^{2}} \frac{\bar{G}_{y z}}{E} H_{m i n j}^{0001} \\
C_{\text {minj }}^{O N}= & -\frac{\tilde{\rho}}{\tilde{I}} \frac{a}{2 \pi^{2}} \frac{\bar{G}_{z x}}{E} H_{m i n j}^{1000} \\
C_{\operatorname{minj}}^{O O}= & \frac{\tilde{\rho}}{\tilde{I}} \frac{c t}{2 \pi^{2}}
\end{aligned}
$$




$$
\begin{aligned}
& \left(\frac{1}{\left(1-\nu^{2}\right)} H_{m i n j}^{1100}+\frac{a^{2}}{2 b^{2}(1+\nu)} H_{m i n j}^{0011}\right) \\
& +\frac{\tilde{\rho}}{\tilde{I}} \frac{a}{4 \pi^{2}} \frac{\bar{G}_{z x}}{E} H_{m i n j}^{0000} \\
& C_{\operatorname{minj}}^{O P}=\frac{\tilde{\rho}}{\tilde{I}} \frac{a c t}{2 b \pi^{2}} \\
& \left(\frac{\nu}{\left(1-\nu^{2}\right)} H_{m i n j}^{0101}+\frac{1}{2(1+\nu)} H_{m i n j}^{1001}\right) \\
& C_{m i n j}^{P N}=-\frac{\tilde{\rho}}{\tilde{I}} \frac{a^{2}}{2 b \pi^{2}} \frac{\bar{G}_{y z}}{E} H_{m i n j}^{0010} \\
& C_{\operatorname{minj}}^{P O}=\frac{\tilde{\rho}}{\tilde{I}} \frac{a c t}{2 b \pi^{2}} \\
& \left(\frac{\nu}{\left(1-\nu^{2}\right)} H_{m i n j}^{1001}+\frac{1}{2(1+\nu)} H_{m i n j}^{0110}\right) \\
& C_{\operatorname{minj}}^{P P}=\frac{\tilde{\rho}}{\tilde{I}} \frac{c t}{2 \pi^{2}} \\
& \left(\frac{a^{2}}{b^{2}\left(1-\nu^{2}\right)} H_{m i n j}^{0011}+\frac{1}{2(1+\nu)} H_{m i n j}^{1100}\right) \\
& +\frac{\tilde{\rho}}{\tilde{I}} \frac{a^{2}}{4 \pi^{2}} \frac{\bar{G}_{y z}}{E} H_{m i n j}^{0000}
\end{aligned}
$$

and

$$
\begin{aligned}
& \tilde{\rho}=2 \rho_{f} t+\rho_{c} c \\
& \tilde{I}=\frac{\rho_{f} c t}{2}+\frac{\rho_{c} c^{2}}{12} \\
& \tilde{f}^{2}=f^{2} \frac{\tilde{\rho} a^{2}}{E} \\
& \omega^{2}=4 \pi^{2} f^{2} \\
& \bar{G}_{z x}=k G_{z x} \\
& \bar{G}_{z y}=k G_{z y}
\end{aligned}
$$

Equation (10) system can be cast in the matricial compact form

$$
\sum_{m=0}^{p-1} \sum_{n=0}^{p-1}\left([K]-\tilde{f}^{2}[M]\right)\{\lambda\}=0
$$

denoting that the formulation results in an eigenvalue problem, where $[K]$ and $[M]$ are stiffness and mass matrixes and $\tilde{f}$ and $\{\lambda\}$ form the eigenvalue-eigenvector pair.

It is worth remarking that damping was not taking into account in this modeling, which, surely, is a limitation of the proposed approach. To diminish this drawback, the damping influence is considered, based on a simplified model, in the experimental results.

\section{Parameter identification}

This section describes the adopted optimization technique to identify elastic constants of the sandwich plate. The technique is based on the adjustment of coefficients in a minimization optimization process with respect to the elastic constants. Based on the least squares method, values are sought for the elastic constants that would provide best agreement between obtained experimental measurements and the analytical model natural frequencies $[3,7,10]$.

As previously mentioned, a sandwich plate composed of a single orthotropic core with two thin isotropic faces was considered. For this sandwich plate, according to the mathematical model described in Section 3, for given plate dimensions and material density, the frequencies are nonlinear functions of the Young's modulus $E$, the Poisson's ratio $\nu$ and the shear moduli $G_{x z}$ and $G_{y z}$. Therefore, to formulate the identification problem the vector $\theta$, which contains the undetermined coefficients, is defined by

$$
\theta=\left[\frac{E}{E_{0}} \nu \frac{G_{x z}}{E_{0}} \frac{G_{y z}}{E_{0}}\right]^{T}
$$

where $E_{0}$ is a fixed scaling factor, chosen so that all parameters are measured on compatible scales.

For identification of elastic constants of thick orthotropic plates, Frederiksen [3,5,6] proposed an objective function based on a sum of squared relative differences between each experimental natural frequency and its corresponding theoretical prediction. Herein the objective function differs a little from Frederiksen's. Motivated by a number of numerical tests, a residue consisting of the difference between the square of experimental and the numerical frequencies was adopted. Therefore, the adopted objective function is written as

$$
L=\sum_{i=1}^{m}\left[e_{i}(\theta)\right]^{2} .
$$

The residue $e_{i}(\theta)$ is defined by

$$
e_{i}(\theta)=\frac{f_{\exp _{i}}^{2}-f_{i}^{2}(\theta)}{f_{\exp _{i}}^{2}}
$$

where $f_{\text {exp }_{i}}$ represents the experimental frequencies and $f_{i}(\theta)$ the numerical ones, obtained in each step of the optimization iterative process. The number of modes $m$ considered in the analysis, depend on the quality of the obtained experimental data and the sensitivity analysis of the problem. The work deals with the first twelve frequencies. Indeed, the above objec- 

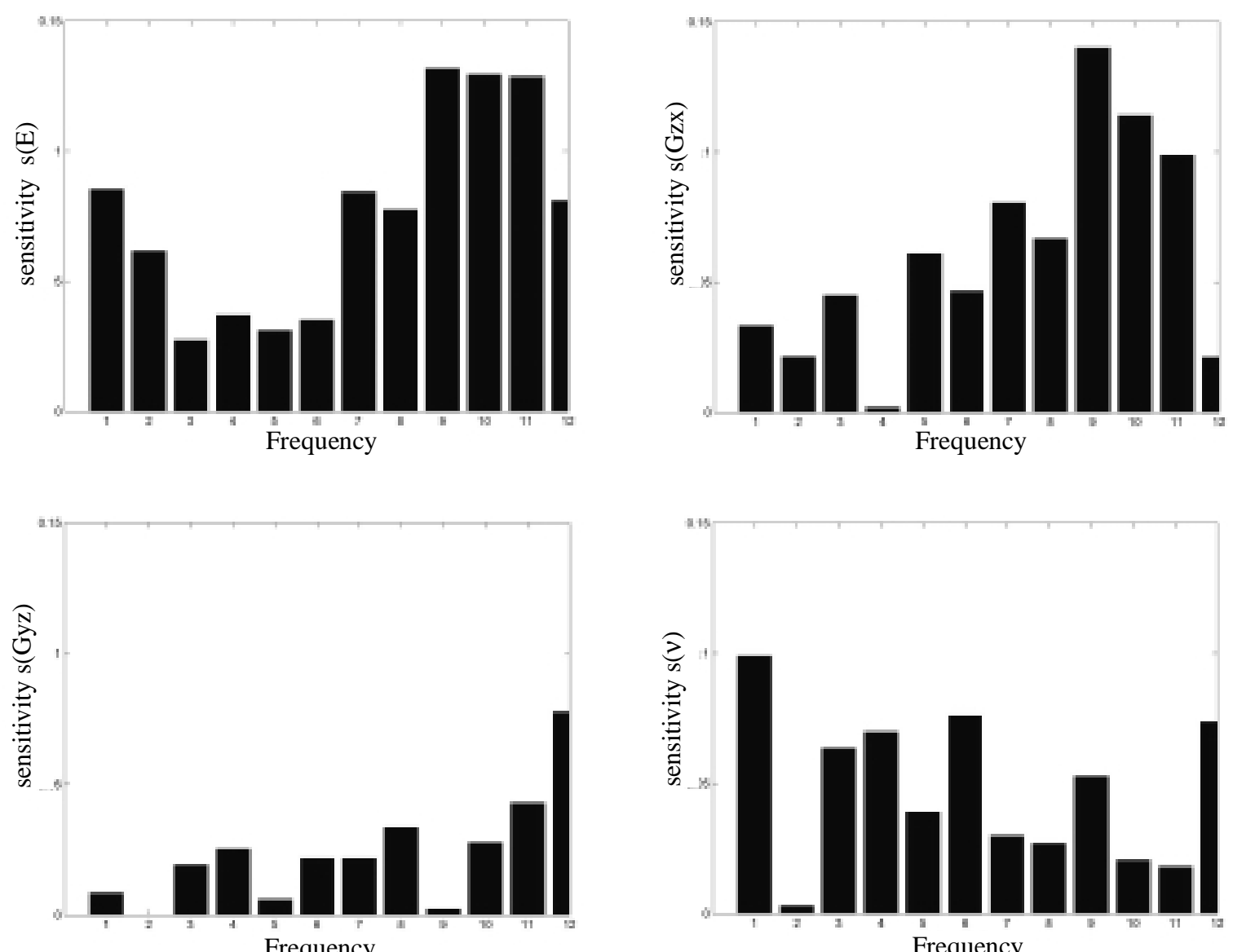

Fig. 4. Sensitivity coefficients for a free edge sandwich plate.

tive function has lead to a better conditioned numerical problem.

In order to fulfill mechanical requirements to ensure the positive-definiteness of the stiffness matrix, the following constraints are imposed to the parameters:

$$
\begin{aligned}
& \frac{E}{E_{0}}>0 \quad \frac{G_{y z}}{E_{0}}>0 \\
& \frac{G_{x z}}{E_{0}}>0-1<\nu<0.5
\end{aligned}
$$

A penalty method is applied to transform this constrained problem into an unconstrained one $[1,2,5]$. In this method, a modified objective function is proposed so as to assign a high cost for violating the constraints. Then, the minimization process is performed iteratively by a Newton-Rapson algorithm. For more details about the algorithm see Bastos [1] and Bazzara [2].

\section{Frequency sensitivity analysis}

First-order derivatives of the frequencies with respect to the unknown parameters, often referred as sensitivities, are primordial in the optimization algorithm based on gradient methods as Newton-like algorithms. Because sensitivity coefficients values indicate the level of the process reliability, more detailed information can be gained for the identification procedure by examining those coefficients [9]. For example, those coefficients are important in order to determine the minimal number of frequencies that need to be included in the analysis to assure reliable results.

The non-dimensional coefficient $s_{i j}(\theta)$, which indicates the sensitivity of the frequency $f_{i}$ with respect to the unknown parameter $\theta_{j}$, is obtained by the analytical expression

$$
s_{i j}(\theta)=\frac{\partial f_{i}(\theta)}{\partial \theta_{j}} \frac{\theta_{j}}{f_{i}(\theta)}
$$


Table 1

Computed $E, G_{y z}, G_{z x}$ and $\nu$ for three different initial guesses

\begin{tabular}{llc}
\hline Initial guess & Identified parameters & Number of iterations \\
\hline$E_{0}=200.00 \mathrm{GPa}$ & $E=59.92 \mathrm{GPa}$ & 25 \\
$G_{0 y z}=0.80 \mathrm{GPa}$ & $G_{y z}=0.43 \mathrm{GPa}$ & \\
$G_{0 z x}=0.20 \mathrm{GPa}$ & $G_{z y}=0.10 \mathrm{GPa}$ & \\
$\nu_{0}=0.40$ & $\nu=0.33$ & \\
\hline$E_{0}=200.00 \mathrm{GPa}$ & $E=59.86 \mathrm{GPa}$ & 25 \\
$G_{0 y z}=1.00 \mathrm{GPa}$ & $G_{y z}=0.43 \mathrm{GPa}$ & \\
$G_{0 z x}=0.40 \mathrm{GPa}$ & $G_{z y}=0.10 \mathrm{GPa}$ & \\
$\nu_{0}=0.20$ & $\nu=0.33$ & \\
\hline$E_{0}=100.00 \mathrm{GPa}$ & $E=59.97 \mathrm{GPa}$ & \\
$G_{0 y z}=0.38 \mathrm{GPa}$ & $G_{y z}=0.42 \mathrm{GPa}$ & \\
$G_{0 z x}=0.1 \mathrm{GPa}$ & $G_{z y}=0.10 \mathrm{GPa}$ & \\
$\nu_{0}=0.30$ & $\nu=0.33$ & \\
\hline
\end{tabular}

where

$$
\frac{\partial f_{i}(\theta)}{\partial \theta_{j}}=\frac{E}{2 \tilde{\rho} a^{2} f_{i}} \frac{\partial K(\theta)}{\partial \theta_{j}} \phi_{i} \cdot \phi_{i}
$$

in which it is assumed that the eigenvector $\phi_{i}$, obtained from the numerical analysis, is normalized such that $[M] \phi_{i} . \phi_{i}=1$.

Figure 4 shows the value of the inverse sensitivity coefficients for a free edge sandwich plate with the following properties: $E=71 \mathrm{GPa}, G_{y z}=0.22 \mathrm{GPa}$, $G_{z x}=0.11 \mathrm{GPa}$ e $\nu=0.30$. The graphs show that, in almost all frequencies range, higher sensitivities with respect to Young's modulus and shear modulus $G_{z x}$ are obtained than those obtained with respect to shear modulus $G_{y z}$ and Poisson's ratio $\nu$. Some precautions should be considered in analyzing the reliability of those parameters estimation. Finally, for all parameters, the graphs indicate that better estimates are possible provided the higher mode frequencies are included.

\section{Results}

Before applying the present methodology to composite structures, it was applied to a homogeneous aluminum plate. This turned out to be a very good test due to the existence of previous estimations of the sought parameters. Besides, there are simpler ways to perform the validation of the updated model. A number of important analysis were developed in this context such as the adequacy of the polynomials, convergence characteristics of the algorithm and strategies for the choice of the initial guess. Those results are reported in [1].

The above sensitivity analysis, summarized in Fig. 4, points towards the use of the 12 first natural frequencies in the identification process. Moreover, for the iterative algorithm used in the optimization, initial guesses are necessary for the sought parameters. The three initial guesses adopted are shown in Table 1. The number of iterations needed is presented as well. Regardless the initial guesses choice the identified parameters are similar, which seems to be an indicator of the consistency and stability of the proposed approach

The shift on the natural frequencies due to the presence of damping was approximated using the expression of a single degree of freedom ("modal damping"). Thus, the damping was evaluated and this was used to obtain normal frequencies from the damped ones.

A typical convergence's history of the optimization is depicted for each parameter in Fig. 5. One can observe that all the elastic parameters converge monotonically in a satisfactory number of iterations. The elastic modulus and the Poisson's ratio achieve the final value much faster than the shear coefficients.

The plate orthotropy [9], due to the core element, renders distinct values for the two shear moduli, as it was experimentally confirmed and presented in Table 1.

\section{Final remarks}

The present work deals with an identification approach, based on model up-date, for global elastic parameters of a composite structure represented by a honeycomb sandwich plate. This approach relies on the comparison of a number of experimental natural frequencies, obtained by means of standard modal tests, with those coming from an analytical modeling. This comparison results in a minimization problem numerically solved by an iterative procedure based on the Newton's method.

Finally, the approach starts with the modal tests execution and finishes with the optimization procedure, in a prototype. The obtained results seem to be satisfactory and, therefore, motivate the authors to apply the same approach to different kinds of composites like, for instance, fiber reinforced laminates. 

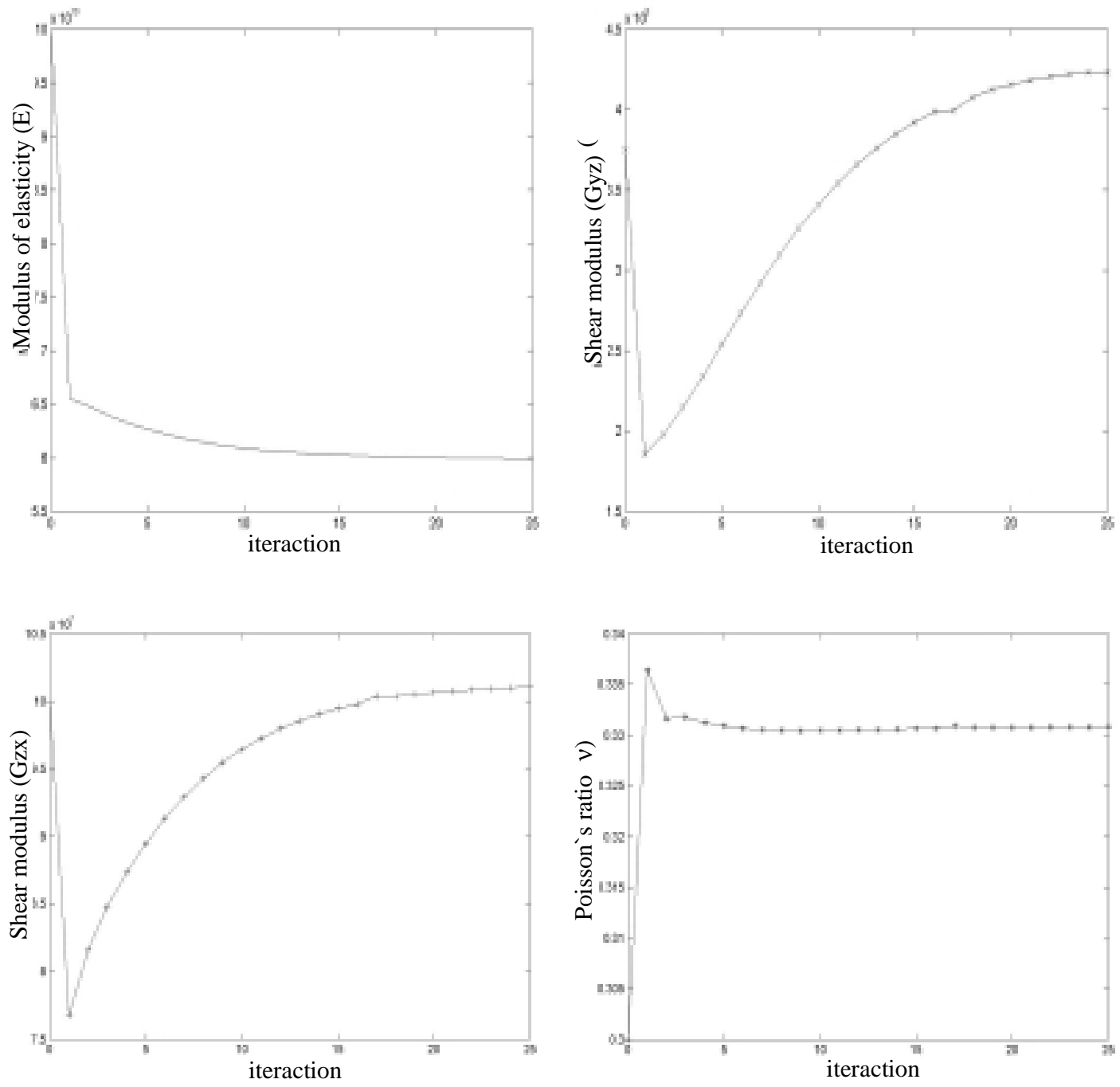

Fig. 5. Parameters evolution along the iterative process.

\section{Acknowledgements}

The authors would like to express their gratitude to Prof. Rubens Sampaio (Vibrations Laboratory /PUCRio) and Engineer José Elias (INPE - National Institute of Aerospace Research) who provided the conditions to performing the experiments. The Brazilian Space Agency (AEB) has, partially, supported the present work under the contract 00403-2000-50/AEB.

\section{References}

[1] S.F. Bastos, A numerical and experimental approach for identifying elastic parameters in sandwich plates. MSc. Disserta- tion. COPPE, Federal University of Rio de Janeiro, Brazil, 2001, in Portuguese.

[2] M.S. Bazaraa and C.M. Shetty, Nonlinear Programming Theory and Algorithms, John Wiley \& Sons, New York, USA, 1979.

[3] S. Frederiksen, Identification of Material Parameters in Anisotropic Plates - A Combined Numerical/Experimental Method. Ph.D Dissertation, The Technical University of Denmark, Hellerup, Denmark, 1992.

[4] S. Frederiksen, Single layer plate theories applied to the flexural vibration of completely fre thick laminates, Journal of Sound and Vibration 186(5) (1995), 743-759.

[5] S. Frederiksen, Numerical studies for the identification of orthotropic elastic constants for thick plates, European Journal of Mechanics. A/Solids 16(1) (1997), 117-140.

[6] S. Frederiksen, Experimental procedure and results for the identification of elastic constants of thick ortrotopic plates, Journal of Composites Material 31(4) (1997), 360-382. 
[7] R.F. Gibson, Modal vibration response measurements for characterization of composite materials and structures, Composite Science and Technology 60 (2000), 2769-2780.

[8] M. Grédiac and P.A. Paris, Direct identification of elastic constants of anisotropic plates by modal analysis: Theoretical and numerical aspects, Journal of Sound and Vibration 195(3) (1996), 401-415.

[9] J.N. Reddy, Mechanics of laminated composite plate, CRC Press, Inc, 1997.

[10] R. Rikards, A. Chate and G. Gailis, Indentification of elestic properties of laminates based on experiment design, Interna- tional Journal of Solids and Structures 38 (2001), 5097-5115.

[11] T. Satto, R.D. Parbery, S. Okuno and S. Kawano, Parameter identification for aluminum honeycomb sandwich panels based on orthotropic Timoshenko beam theory, Journal of Sound and Vibration 268(2) (1997), 271-287.

[12] C.Y. Son, I.T. Kim and J.S. Paik, An experimental study on behavior and vibrational characteristics of sandwich plate with aluminum honeycomb core, Proceedings of the Seventh International Offshore and Polar Engineering Conference, Honolulu, USA, 1997, pp. 697-703. 

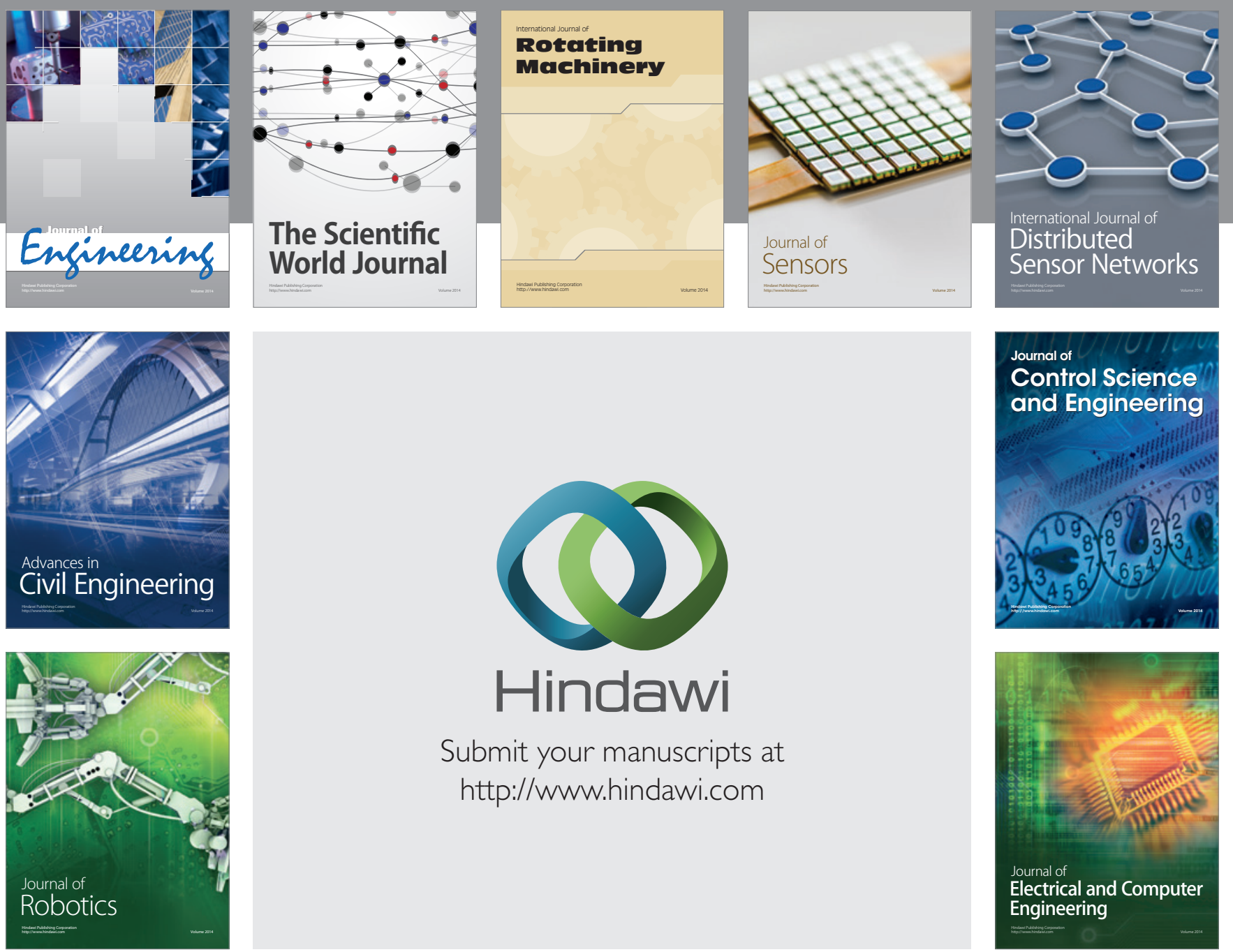

Submit your manuscripts at

http://www.hindawi.com
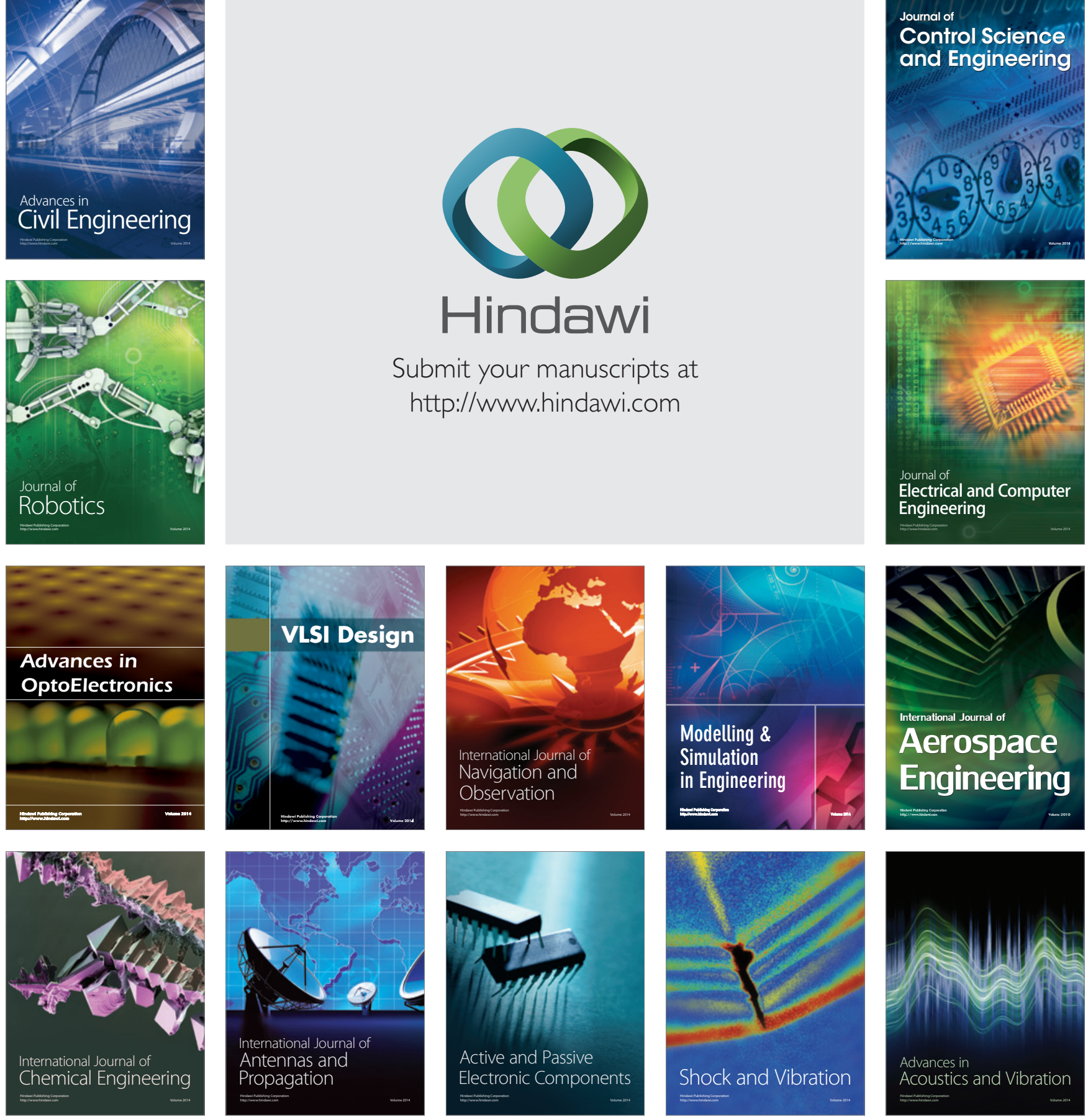